\title{
Gangrène des organes génitaux externes (GOGE) : traitement chirurgical et reconstruction plastique
}

\section{Gangrene of male external genitalia: surgical treatment and plastic reconstruction}

\author{
A. Dekou - P.-G. Konan - E. Gowe $\cdot$ C. Vodi - B. Kouame $\cdot$ A. Fofana - G.-A. Ouegnin $\cdot$ K. Manzan \\ Reçu le 8 juin 2011 ; accepté le 26 septembre 2011 \\ (C) SALF et Springer-Verlag France 2011
}

Résumé Buts : Présenter notre contribution à la reconstruction chirurgicale de la gangrène des organes génitaux externes (GOGE), selon les lésions.

Matériel et méthodes : Il s'agit d'une étude rétroprospective, portant sur 14 patients traités et guéris dans le service d'urologie du CHU de Cocody à Abidjan, de GOGE entre janvier 2000 et juin 2009.

Résultats : L'âge moyen des patients était de 39 (20-67) ans. Tous étaient des hommes de race noire. Ils présentaient tous un tableau infectieux traité par une réanimation hydroélectrolytique et une triantibiothérapie. Les lésions cutanées observées intéressaient les organes génitaux externes (OGE) dans neuf cas $(64,29 \%$ ), puis le scrotum et le périnée dans cinq cas $(35,71 \%)$. Toutes les lésions cutanées ont été décapées, suivies de pansements locaux. Ainsi, 14 patients $(100 \%)$ ont été guéris, complètement cicatrisés, dont trois cas $(21,43 \%)$ de lésions scrotales minimes qui ont cicatrisé spontanément à la suite de pansement uniquement, neuf patients $(64,29 \%)$ ont cicatrisé à la suite de suture sans tension de la peau scrotale après débridement, décollement, enfin deux patients $(14,28 \%)$ avaient cicatrisé après une greffe de peau mince prélevée à la face interne de la cuisse pour plaie périnéopénoscrotale étendue, d'une part, et, d'autre part, pour une perte complète du fourreau pénien.

Conclusion : La GOGE est une affection grave, qui réalise, d'une part, une toxi-infection traitée par une réanimation et une triantibiothérapie, d'autre part, des lésions cutanées plus ou moins étendues nécessitant débridement, décapage, pansement, puis une reconstruction chirurgicale plastique qui restaure un environnement pénoscrotal adéquat qui ne compromette pas la qualité des rapports sexuels ainsi que la fonc-

\footnotetext{
A. Dekou $(\bowtie) \cdot$ P.-G. Konan $\cdot$ E. Gowe $\cdot$ C. Vodi $\cdot$ B. Kouame

A. Fofana · G.-A. Ouegnin · K. Manzan

Service d'urologie, CHU Cocody, BPV 13 Abidjan,

Côte-d'Ivoire

e-mail : dekouangoran@yahoo.fr
}

tion de reproduction des testicules, surtout chez le sujet jeune. Pour citer cette revue : Andrologie 21 (2011).

Mots clés Gangrènes · Organes génitaux externes ·

Traitement $\cdot$ Chirurgie plastique

\begin{abstract}
Objective: To present the various types of surgical reconstruction of Fournier's gangrene and their outcome. Patients and methods: It is a retrospective study conducted on 14 patients with Fournier's gangrene treated at the Urology Department of Teaching Hospital of Cocody (Abidjan-Côte d'Ivoire) between January 2000 and June 2009. Results: The mean age of the patients was 39 years (range: 20-67 years). All the patients were black and presented infectious symptoms, which were treated by hydroelectrolytic reanimation and ant biotherapy. The lesions reached down all the penis and the scrotum in $64.29 \%$ of the cases $(N=9)$ and on the scrotum and perinea in $35.71 \%$ of the cases $(N=5)$. The lesions require extensive debridement and local bandages. All of the patients were cured $(100 \%)$. In three patients $(21.43 \%)$, the lesions with bandages only to tiny scrotum lesions healed spontaneously. In nine patients $(64.29 \%)$, it got healed after debridement suture without tension of the scrotum skin, and in the two patients $(14,28 \%)$, the one with wide wound of penis, scotum and perinea, then the other with penile skin loss, were healed after skin graft taken with inner face of the thigh.

Conclusion: Fourniers gangrene still remains a severe disease. Management of this gangrene requires a multidisciplinary approach. The plastic surgical reconstruction restores an adequate environment to penis and scrotum, which does not compromise the quality of the sexual relations and the reproductive function of the testicles, especially in young men. To cite this journal: Andrologie 21 (2011).
\end{abstract}

Keywords Gangrene - Male external genitalia - Treatment Plastic surgery 


\section{Introduction}

La gangrène des organes génitaux externes (GOGE), appelée également gangrène de Fournier, correspond à une fasciite nécrosante de la région périnéale qui touche majoritairement les hommes de tous âges, exceptionnellement les femmes [1-5].

Le traitement de la gangrène de Fournier doit être multidisciplinaire, instauré en extrême urgence et réalisé sous surveillance étroite dans une unité de soins intensifs. Ce traitement est médical d'une part, avec administration d'une large antibiothérapie, et chirurgical d'autre part, avec un débridement complet des tissus nécrosés $[1,3,5]$.

Cette prise en charge thérapeutique multidisciplinaire peut être associée à une reconstruction chirurgicale à distance $[1,5,6]$.

Le but de cette étude est de présenter notre contribution à la reconstruction chirurgicale de la GOGE, selon l'importance des lésions, afin de restaurer un environnement adéquat qui ne compromette pas la qualité des rapports sexuels ainsi que la fonction de reproduction des testicules.

\section{Patients et méthodes}

Il s'agit d'une étude rétro- et prospective qui a colligé 20 dossiers de patients qui ont présenté une gangrène périnéoscrotale et dont 14 ont été retenus dans notre étude. Ces malades étaient hospitalisés dans le service d'urologie du CHU de Cocody à Abidjan (Côte-d'Ivoire), entre janvier 2000 et juin 2009, et étaient tous des hommes de race noire.

\section{Critères d'inclusion}

Tous les patients vivants, qui ont présenté une gangrène périnéoscrotale, avec un index de sévérité de la gangrène de Fournier $($ FGSI $<9)$ et après avoir subi toutes les étapes du traitement : réanimation hydroélectrolytique, débridement cutané, pansement jusqu'à bourgeonnement de la plaie, cicatrisation spontanée ou reconstruction plastique, puis guérison définitive avec des dossiers comportant les paramètres étudiés, ont été inclus dans l'étude (14 patients).

\section{Critères de non-inclusion}

Tous les patients ayant souffert de GOGE et décédés en cours de réanimation, avec état général très altéré et désordre métabolique important (FGSI > 9) [six cas].

\section{Paramètres étudiés}

- l'épidémiologie : notamment, l'âge, le délai d'admission, la durée d'hospitalisation, les étiologies ;
- la clinique : telle, les antécédents (ATCD) médicaux, les signes cliniques, la description des lésions, ainsi que les affections associées, avec une notion d'alcoolisme chronique (durée moyenne de consommation de neuf ans [6-18]) chez quatre patients (dépendance au vin rouge et aux liqueurs), de quantité quotidienne non précisée ;

- les examens complémentaires : précisément, la numération formule sanguine, le ionogramme sanguin, la créatininémie, les prélèvements bactériologiques des plaies, la sérologie VIH et la glycémie. L'urétrocystographie rétrograde (UCR) avait été demandée par la suite pour les patients présentant une obstruction urétroprostatique. Aucune hémoculture n'a pu être réalisée.

\section{Traitements réalisés}

\section{Traitement médical}

Il était institué selon le schéma suivant :

- l'antibiothérapie par voie parentérale d'emblée plus ou moins adaptée à l'antibiogramme jusqu'à l'amélioration de l'état général du malade (chute de la fièvre, reprise de l'alimentation normale), puis relais par voie orale pendant 14 à 21 jours selon l'évolution clinique.

L'antibiothérapie parentérale consistait en l'association le plus souvent de trois antibiotiques : une bêtalactamine en particulier les céphalosporines de troisième génération ( 2 à $4 \mathrm{~g} / \mathrm{j}$ jusqu'à la chute de la fièvre), un aminoside (150 à $300 \mathrm{mg} / \mathrm{j}$ pendant sept jours) et un métronidazole (1,5 g/j pendant dix jours). L'antibiothérapie encadrait le plus souvent les gestes chirurgicaux ;

- une réanimation médicale faite de la rééquilibration hydroélectrolytique, parfois de transfusion sanguine isogroupe isorhésus ;

- à ce protocole étaient associés, en fonction des comorbidités, une insulinothérapie (en cas de diabète), une trithérapie antirétrovirale (VIH) et un traitement antihypertenseur (en cas d'HTA).

\begin{tabular}{|lll|}
\hline \multicolumn{2}{|l|}{ Tableau 1 Antibiothérapie parentérale utilisée } \\
\hline $\begin{array}{l}\text { Association d'antibiotiques } \\
\text { utilisés }\end{array}$ & Effectifs & Pourcentage \\
\hline $\begin{array}{l}\text { Ceftriaxone + nétromicine }+ \\
\text { métronidazole }\end{array}$ & 11 & 78,6 \\
$\begin{array}{l}\text { Cefotaxime + tobramycine }+ \\
\text { métronidazole }\end{array}$ & 3 & 21,4 \\
Total & 14 & 100 \\
\hline
\end{tabular}


L'antibiothérapie parentérale était utilisée selon les associations indiquées dans le Tableau 1.

\section{Traitement chirurgical}

Il a consisté en :

- l'évacuation des collections purulentes et à l'excision de tous les tissus nécrosés (Fig. 1). Tous nos patients ont bénéficié de traitement chirurgical. Ce traitement a été réalisé sous anesthésie générale. Il a consisté en une mise à plat avec excision des téguments et de tous les tissus nécrosés. Des exérèses complémentaires de nouvelles zones nécrotiques ont été entreprises dans quatre cas, quatre cystostomies ont été réalisées pour sténose urétrale ;

- les soins locaux : pansement journalier à la solution de Dakin ou bain de siège au Dakin pendant sept à dix jours selon l'importance, le siège et l'aspect des lésions, associé à une toilette à l'eau oxygénée, puis si la plaie est propre, nous poursuivons le pansement au tulle gras tous les trois à quatre jours jusqu'à obtention d'une plaie prête pour la reconstruction;

- la reconstruction plastique était réalisée lorsque les plaies étaient propres, bien bourgeonnées :

- si la lésion est moins étendue (Fig. 2), nous réalisons un décollement-avivement-suture cutané des berges. Parfois, une cicatrisation spontanée par pansement suffit (Fig. 3) ;

- si la lésion est étendue (Figs. 4 et 5), nous réalisons une greffe de peau mince ;

- quant aux patients qui ont présenté une gangrène sur rétention d'urines par sténose de l'urètre, une dérivation

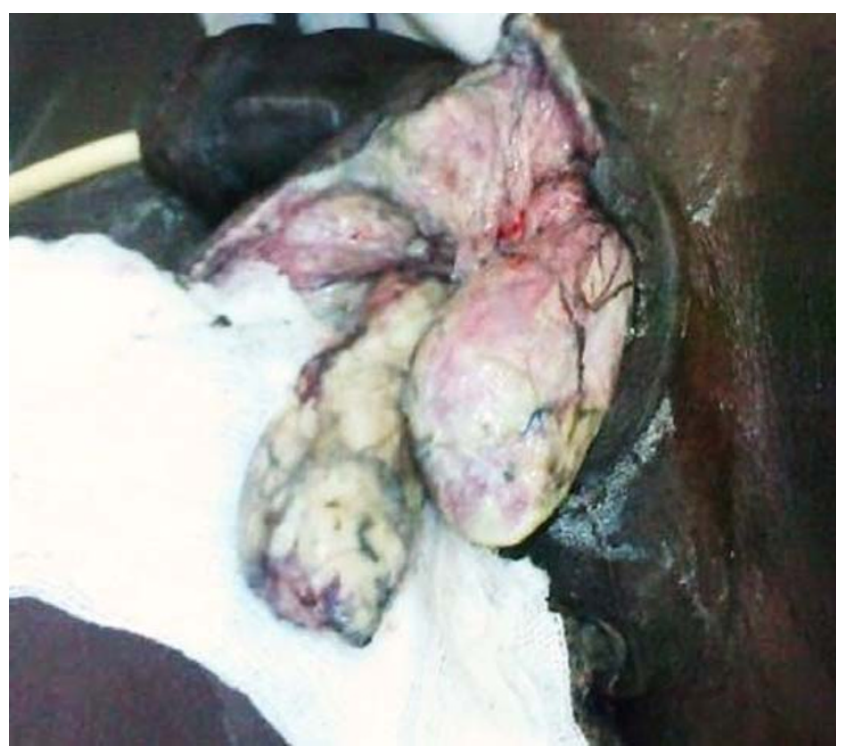

Fig. 1 GOGE : excision et débridement des tissus nécrotiques (j2 postopératoire) urinaire par cystostomie a été associée, puis une cure du rétrécissement effectuée à distance, après cicatrisation complète de la reconstruction plastique.

\section{Résultats}

\section{Aspects épidémiologiques}

- L'âge : la GOGE a atteint les hommes adultes avec un âge moyen de 39,4 [20-67] ans, dont 64,29\% avait moins de 50 ans ;

- les étiologies : les gangrènes étaient consécutives à des affections urologiques et proctologiques, enfin dans

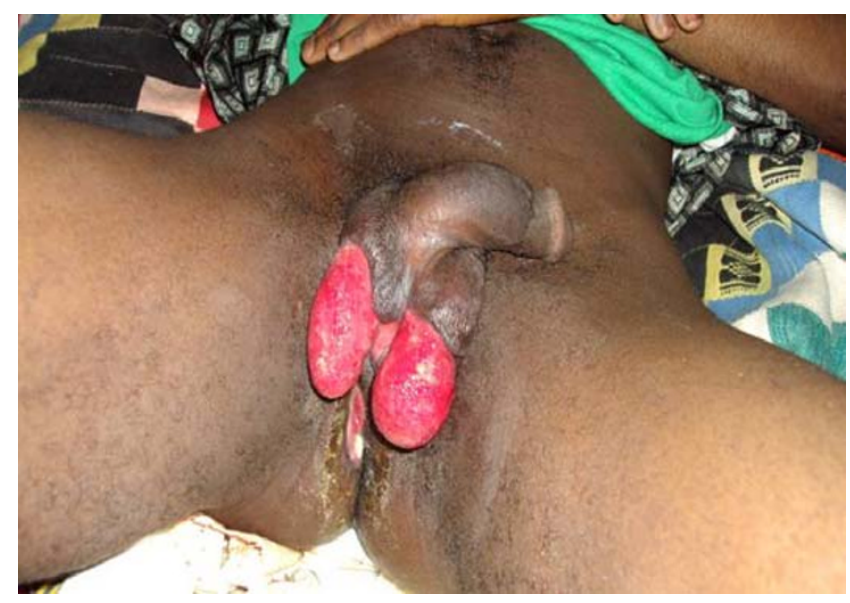

Fig. 2 GOGE consécutive à un abcès anal, ayant évolué favorablement après excision et pansement quotidien (j14 postopératoire)

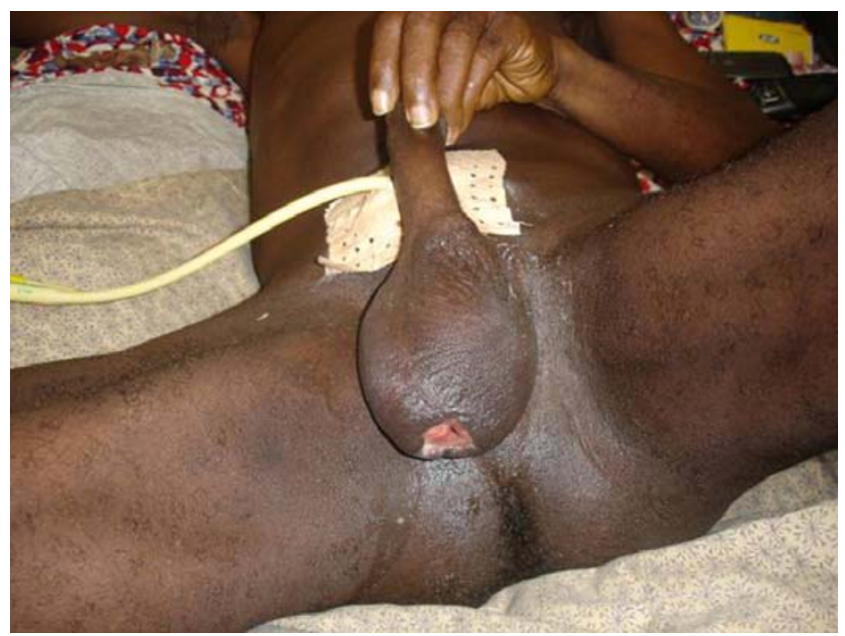

Fig. 3 GOGE de cause urologique évoluant favorablement sous dérivation urinaire et pansement ( $\mathrm{j} 11$ postopératoire) 


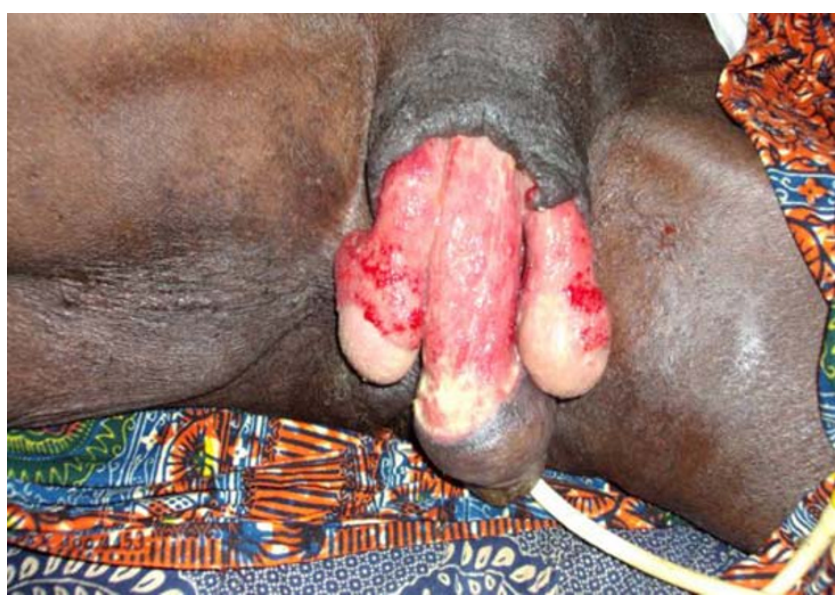

Fig. 4 GOGE chez un diabétique (idiopathique). Excision cutanée plus pansement quotidien ( 17 postopératoire)

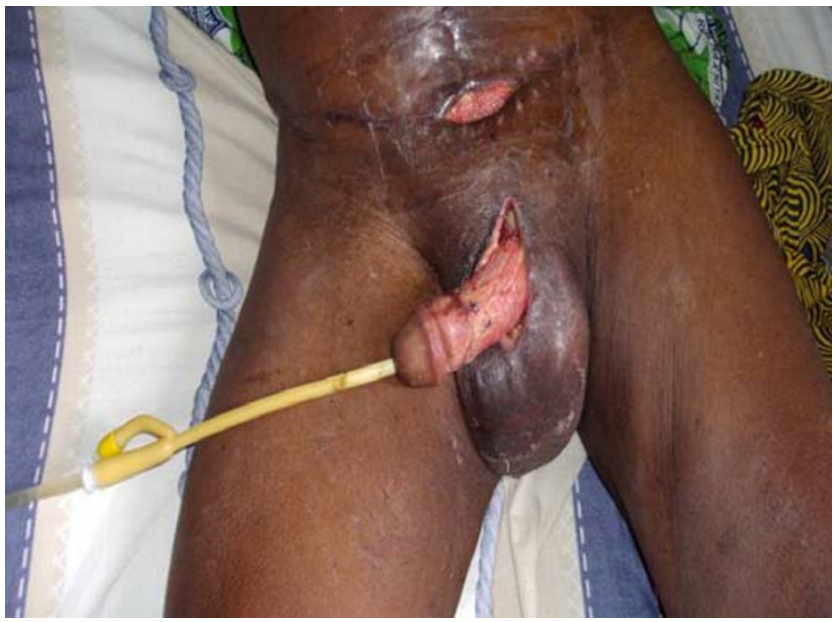

Fig. 5 GOGE iatrogène (j12 postopératoire)

$58 \%$ des cas, aucune étiologie n'a été retrouvée telle que présentée dans le Tableau 2.

\section{Aspects diagnostiques}

\section{Délai de consultation et durée d'hospitalisation}

Le délai moyen de consultation des patients était de 7,40 jours (extrêmes : 3 et 16).

La durée d'hospitalisation était variable, entre 18 et 102 jours, avec une moyenne de 32 jours.

\section{Antécédents médicaux}

Cinq cas, soit $35,71 \%$ des patients, portaient des maladies chroniques telles que présentées dans le Tableau 3.
En définitive, les patients sont répartis en fonction des étiologies et des antécédents médicaux comme présentés dans le Tableau 4.

\section{Signes cliniques}

La totalité des malades avait présenté des douleurs scrotales, un syndrome infectieux et des nécroses cutanées. Les différents signes sont répertoriés dans le Tableau 5.

\section{Siège des lésions}

Les lésions intéressaient les OGE dans $45 \%$ des cas, puis le scrotum et le périnée dans $55 \%$ des cas dont un cas avec extension à l'aine et aux fesses.

\begin{tabular}{|lll|}
\hline Tableau 2 & Répartition des patients en fonction des étiologies \\
\hline Causes probables & Effectifs & Pourcentage \\
\hline Aucune (idiopathique) & 8 & 58 \\
Sténose urétrale & 3 & 21 \\
Abcès anal & 1 & 7 \\
Hémorroïdes & 1 & 7 \\
Iatrogène & 1 & 7 \\
Total & 14 & 100 \\
\hline
\end{tabular}

Tableau 3 Répartition des patients selon les antécédents médicaux

\begin{tabular}{|lll|}
\hline Antécédents & Effectifs & Pourcentage \\
\hline Diabète & 2 & 14,29 \\
Infection à VIH & 1 & 7,14 \\
Drépanocytose AS & 1 & 7,14 \\
Adénocarcinome & 1 & 7,14 \\
de la prostate & & \\
Aucun & 9 & 64,29 \\
Total & 14 & 100 \\
\hline
\end{tabular}

Tableau 4 Répartition des patients en fonction des étiologies et des antécédents médicaux

\begin{tabular}{|lll|}
\hline Comorbidité & Effectifs & Pourcentage \\
\hline Idiopathique & 8 & 58 \\
Sténose urétrale + drépanocytose & 1 & 7 \\
Sténose urétrale + VIH + alcool & 1 & 7 \\
Sténose urétrale + diabète + alcool & 1 & 7 \\
Abcès anal + diabète + alcool & 1 & 7 \\
Hémorroïde + adénocarcinome & 1 & 7 \\
de la prostate + alcool & & \\
Iatrogène & 1 & 7 \\
Total & 14 & 100 \\
\hline
\end{tabular}


Tableau 5 Récapitulatif des signes cliniques locaux et généraux en fonction des malades

\begin{tabular}{|lll|}
\hline Manifestations cliniques & Effectifs & Pourcentage \\
\hline Douleurs des bourses + & 14 & 100 \\
syndrome infectieux & & \\
Plage de nécroses cutanées & 14 & 100 \\
Collections et sécrétions purulentes & 10 & 71,4 \\
Edèmes et phlyctènes & 5 & 35,7 \\
\hline
\end{tabular}

\section{Données biologiques}

Soixante-dix-huit pour cent des patients (11 cas) avaient une hyperleucocytose supérieure à 10000 [10 000$17000]$ elts $/ \mathrm{mm}^{3}$.

Soixante et onze pour cent (dix cas) étaient anémiés avec un taux moyen d'hémoglobine de 9,1 [4,9-11,9] g/dl.

Un malade sur 14 , soit 7,14\%, était séropositif.

L'examen cytobactériologique du pus n'a pu être prélevé que chez cinq patients $(35,71 \%)$. Les germes isolés étaient Klebsiella pneumoniae (un cas), Levinea (un cas) et Staphylococcus aureus associé à Pseudomonas aeroginosa (un cas). Les deux autres examens étaient négatifs.

\section{Aspects thérapeutiques}

\section{Traitement médical}

Le traitement médical par triantibiothérapie et réanimation hydroélectrolytique a permis de traiter efficacement le syndrome infectieux chez tous nos patients ; quatre patients $(28,57 \%)$ avec une anémie de moins $7 \mathrm{~g} / \mathrm{dl}$ ont eu une transfusion sanguine isogroupe, isorhésus. Tout cela a permis une bonne récupération de l'état général et a contribué à une bonne évolution des lésions sous pansement, favorisant ainsi la reconstruction chirurgicale qui a donné $100 \%$ de bons résultats en termes de cicatrisation effective et de guérison totale telles que présenté comme suit.

\section{Traitement chirurgical et résultats}

Quatorze patients ont été guéris tels que :

- trois patients $(21,42 \%)$ ont cicatrisé spontanément à la suite de pansement uniquement pour lésion scrotale minime ;

- neuf patients $(64,29 \%)$ ont cicatrisé à la suite de suture de la peau scrotale après débridement, décollement dont deux cas de reprise pour lâchage secondaire, pour plaie localisée peu étendue ;

- deux patients $(14,29 \%)$ avaient cicatrisé après une greffe de peau prélevée à la face interne de la cuisse pour plaie périnéopénoscrotale étendue, d'une part, et, d'autre part, pour une lésion pénienne.

\section{Discussion}

\section{Aspects épidémiologiques}

Dans notre série, la GOGE atteint les adultes jeunes de plus de 20 ans avec un âge moyen de 39 ans, au contraire des auteurs maghrébins, occidentaux et japonais qui rapportent un âge moyen de 55 à 59 ans [1,2,5,7-9]. Le délai d'admission était par ailleurs plus long dans notre étude que dans les séries occidentales et japonaises. Ces différences s'expliqueraient par la différence des niveaux socio-économiques et culturels. En effet, tous nos patients vivaient dans des conditions sociales et économiques précaires et plus de la moitié était admise avec un retard de plus d'une semaine après le début des symptômes.

L'analyse des antécédents des malades trouve le plus souvent des facteurs associés qui favoriseraient l'infection et la constitution de la gangrène $[5,10,11]$. Ces facteurs étaient, dans notre travail, l'infection à VIH, mais principalement le diabète et l'alcoolisme. Des auteurs ont également rapporté ces mêmes facteurs et la corticothérapie, qui agissent en entraînant un état de diminution des défenses immunitaires et des thromboses vasculaires [3,10-14]. Comme le rapportent également Vanden-Bempt et al. [15], l'Actinobaculum schaali, un micro-organisme, serait également responsable des GOGE. Hormis ces affections intercurrentes, les GOGE ont été provoquées dans notre étude, d'une part, par des sténoses urétrales, d'autre part, par des abcès anorectaux et enfin par une cure chirurgicale simultanée d'un adénome de la prostate associé à une sténose urétrale chez un patient âgé de 64 ans. Dans $58 \%$ des cas, aucune étiologie et aucun facteur favorisant n'ont pu être retrouvés. Dans cette série, la GOGE était, dans $43 \%$ des cas, secondaire à une cause ou sur un terrain morbide identifié qu'il faut absolument rechercher devant toute gangrène pelvipérinéale ; ces causes sont souvent rapportées dans la littérature $[2,4,11]$. Cependant, nous pensons que les causes sont nombreuses, mais parfois inavouées telles chez les patients jeunes (sodomie), ou passées inaperçues par défaut d'investigation (traumatisme minime ou dermatose négligée des OGE). Devant une GOGE, une cause doit être recherchée.

Ainsi donc, la GOGE décrite comme étant idiopathique (maladie de Fournier) dans les anciennes études apparaît de plus en plus aujourd'hui dans presque toutes les études récentes comme étant une gangrène à prédominance secondaire à une cause urogénitale, anorectale, dermatologique, post-traumatique ou postopératoire [7-18]. Idiopathique ou secondaire, la GOGE ou maladie de Fournier est, de notre 
point de vue, une et identique maladie avec le même schéma thérapeutique, comme le rapportent également d'autres auteurs [17-19].

\section{Aspects diagnostiques}

Le diagnostic de la GOGE est confirmé essentiellement par l'examen clinique, cependant en cas de doute, on a recours à l'échographie scrotale afin de confirmer le diagnostic selon certains auteurs [19]. L'imagerie par résonnance magnétique permet de dresser avec précision le bilan d'extension de la gangrène et de préciser son point de départ $[19,20]$. Dans notre série, nous n'avions jamais eu recours à l'imagerie, tant le contexte clinique était évident, comme le rapportent d'autres auteurs $[5,6]$. Tous nos patients ont été vus au stade de gangrène évoluée, évidente à l'inspection. Les douleurs et nécroses des bourses ainsi que le syndrome infectieux étaient présents chez tous nos malades. Cependant, les huit patients avec gangrène idiopathique avaient des lésions cutanées moins étendues, dont quatre ne présentaient pas de collection purulente. La gangrène secondaire aux sténoses urétrales a une particularité, elle commence par une infiltration d'urines dans le tissu cellulograisseux sous-cutané pelvipérinéal qui se résorbe facilement dans les cas précoces, après la pose d'un cathéter sus-pubien, et donne souvent des lésions moins étendues.

\section{Données biologiques}

Différentes bactéries ont été isolées à l'examen cytobactériologique du pus prélevé chez nos patients. Il s'agissait dans la majorité des cas de bacille Gram négatif et de cocci Gram positif. Les bactéries fréquemment retrouvées dans la littérature sont l'Escherichia coli, les bactéroïdes, le $S$. aureus, Proteus, P. aeroginosa et les Enterobacter $[8,12,13]$. Certains de nos patients avaient une anémie sévère, corrigée par une transfusion. Les autres fonctions vitales étaient très peu perturbées.

\section{Aspects thérapeutiques}

La prise en charge thérapeutique des GOGE associe l'excision des tissus nécrosés, la réanimation et l'antibiothérapie probabiliste à large spectre administrée dès l'admission des malades $[8,17,18]$. Il s'agit d'une large excision emportant tous les tissus nécrosés jusqu'en zone saine [18,21]. Cette attitude radicale a pour avantage de freiner l'évolution des lésions et de limiter le nombre de reprise chirurgicale, sous anesthésie générale. Pour certains auteurs, l'excision devrait être économe et limitée aux tissus franchement nécrotiques pour permettre à des îlots cutanés périnéaux une régénération ultérieure sans greffe secondaire [21,22].
La prise en charge chirurgicale de nos malades a été agressive avec excision complète de toutes les surfaces cutanées nécrosées au niveau du scrotum et du périnée, emportant tous les tissus dévitalisés et les zones sphacélées jusqu'en zone hémorragique. Les zones non nécrosées mais infectées étaient traitées de façon moins agressive par de larges incisions de décharge avec drainage par des lames de Delbet. À ces incisions était associé un drainage urinaire qui avait pour but l'assèchement des lésions périnéales. Le sondage vésical a été pratiqué chez $71 \%$ de nos patients, aussitôt l'antibiothérapie et la réanimation instaurées. Pour certains auteurs, la dérivation devrait se faire par cystocathéter pour éviter le risque d'infection ascendante lors des sondages urétraux [23]. Les patients $(29 \%)$ avec une GOGE consécutive à une sténose urétrale ont bénéficié d'emblée d'une cystostomie.

Nous n'avons jamais eu recours à la colostomie, comme le préconisent certains auteurs, pour éviter les souillures des plaies périnéales. En effet, d'un auteur à un autre, les lésions, le traitement, les résultats sont variables et souvent peu comparables car les séries sont également variables et souvent de petites tailles, du fait que la GOGE reste une maladie rare.

L'antibiothérapie réalisée était le plus souvent administrée en triple association [3-24]. L'association la plus utilisée dans notre étude était une céphalosporine de troisième génération, un aminoside et un imidazolé. En effet, les bactéries habituellement isolées dans les GOGE sont régulièrement sensibles à cette association $[8,17]$. Il s'agit d'une association initiale qui pourrait être adaptée ou modifiée en fonction de l'évolution clinique et des données bactériologiques.

La reconstruction plastique dans notre travail s'est faite lorsque la plaie était propre, avec un bourgeonnement affleurant à peine les berges cutanées en évitant des tissus rétractiles de mauvaise qualité, témoins d'une cicatrisation dirigée trop poussée de longue durée. Tous les décollements cutanés étaient sans tension (neuf cas), et ont fait l'objet d'une suture simple par rapprochement des berges cutanées. Une greffe par prélèvement de peau mince à la face antéro-interne de la cuisse a permis de recouvrir un cas de lésion étendue périnéoscrotale et un autre cas de lésion pénienne ; dans ces derniers cas, la couverture cutanée pénienne a nécessité le prélèvement de peau glabre.

Certains traitements adjuvants, tels que l'oxygénothérapie hyperbare et le miel, donnant de bons résultats selon certains auteurs [7,14,24], n'ont pas été utilisés dans notre service. Nos patients avaient, certes, une fréquence respiratoire modérément augmentée, mais n'avaient pas une détresse respiratoire ayant nécessité une oxygénothérapie.

$\mathrm{Au}$ plan pronostique, les résultats du traitement médicochirurgicoplastique ont été satisfaisants du fait d'une prise en charge multidisciplinaire des patients dont les affections comorbides et les étiologies ont été correctement prises en charge. Ce bon résultat dans notre série est d'abord lié à la sélection des malades dont l'index de sévérité de la gangrène 
était inférieur ou égal à 9, et comme le rapportent la plupart des auteurs [7,25], la probabilité de survie des patients est très élevée, supérieure à $80 \%$, lorsque le score est inférieur ou égal à 9 . Ce bon résultat est également lié à la jeunesse des patients ainsi qu'à une consultation précoce.

Ainsi, nous avons volontairement omis les dossiers de tous les malades décédés (qui font l'objet d'une autre étude). Le but de cette sélection était d'homogénéiser et d'harmoniser notre série enfin de ressortir les caractéristiques épidémiologiques, diagnostiques, thérapeutiques et évolutives des patients vivants. Ces caractéristiques dans cette étude étant la jeunesse des patients, la précocité de la prise en charge de la gangrène, les désordres hémodynamiques et polyviscéraux peu importants et la guérison définitive.

En effet, si Fournier, en 1883, a décrit à partir de cinq cas cette gangrène comme foudroyante et idiopathique, chez des patients jeunes [3], quel est le profil actuel des patients qui souffrent de gangrène foudroyante, mortelle, en particulier en Côte-d'Ivoire, malgré le progrès de la réanimation et l'utilisation de l'antibiotique découvert en 1928 par Alexandre Fleming [26], dans le traitement des GOGE ? Cette question motive notre équipe à travailler dans une étude en cours sur « la mortalité dans les gangrènes de Fournier : les facteurs de mauvais pronostic », surtout que la littérature mondiale rapporte dans cette affection un taux de mortalité élevé entre 12 et $45 \%$ [1-25], ce qui explique le recrutement de nos patients, tous vivants dans la présente étude.

\section{Conclusion}

La GOGE est une affection grave, qui réalise d'une part une toxi-infection traitée par une réanimation et une triantibiothérapie, d'autre part, des lésions cutanées plus ou moins étendues nécessitant une reconstruction chirurgicale plastique. Les sutures chirurgicales scrotales se font sans tension et la greffe réalisée avec une peau mince, de sorte à restaurer un environnement pénoscrotal adéquat qui ne compromette pas la qualité des rapports sexuels ainsi que la fonction de reproduction des testicules, surtout chez le patient jeune.

Conflit d'intérêt : les auteurs déclarent ne pas avoir de conflit d'intérêt.

\section{Références}

1. Czymek R, Hildebrand P, Kleemann M, et al (2009) New insights into the epidemiology and etiology of Fournier's gangrene: a review of 33 patients. Infection 37:306-12

2. Koukouras D, Kallidonis P, Panagopoulos C, et al (2011) Fournier's gangrene, a urologic and surgical emergency: presentation of a multi-institutional experience with 45 cases. Urol Int 86:167-72

3. Sehmi S, Osaghae S (2011) Type II diabetes mellitus: new presentation manifesting as Fournier's gangrene. JRSM Short Rep 2:51
4. Sözener U, Gedik E, Kessaf Aslar A, et al (2011) Does adjuvant antibiotic treatment after drainage of anorectal abscess prevent development of anal fistulas? A randomized, placebo-controlled, double-blind, multicenter study. Dis Colon Rectum 54:923-9

5. Sarkis P, Farran F, Khoury R, et al (2009) Gangrène de Fournier : revue de la littérature récente. Prog Urol 19:75-84

6. Eke N (2000) Fournier's gangrene: a review of 1,726 cases. Br J Surg 87:718-28

7. Corcoran AT, Smaldone MC, Gibbons EP, et al (2008) Validation of the Fournier's gangrene severity index in a large contemporary series. J Urol 180:944-8

8. Anchi T, Tamura K, Inoue K, et al (2009) Experience in the treatment of eight cases of Fournier's gangrene. Hinyokika Kiyo $55: 545-9$

9. Luján Marco S, Budía A, Di Capua C, et al (2010) Evaluation of a severity score to predict the prognosis of Fournier's gangrene. BJU Int 106:373-6

10. Picramenos D, Deliveliotis C, Macrichoritis K, Alexopoulou K (1995) La gangrène de Fournier : étiologie, traitements et complications. Prog Urol 5:701-5

11. Mills MK, Faraklas I, Davis C, et al (2010) Outcomes from treatment of necrotizing soft-tissue infections: results from the National Surgical Quality Improvement Program database. Am J Surg 200:790-6

12. Kabay S, Yucel M, Yaylak F, et al (2008) The clinical features of Fournier's gangrene and the predictivity of Fournier's gangrene severity index on the outcomes. Int Urol Nephrol 40:997-1004

13. Ghnnam WM (2008) Fournier's gangrene in Mansoura, Egypt: a review of 74 cases. J Postgrad Med 54:106-9

14. Quatan N, Kirby RS (2004) Improving outcomes in Fournier's gangrene. BJU Int 93:691-2

15. Vanden-Bempt I, Van Trappen S, Cleenwerck I, et al (2011) Actinobaculum schaalii causing Fournier's gangrene. J Clin Microbiol 49:2369-71

16. Ayan F, Sunamak O, Paksoy SM, et al (2005) Fournier's gangrene: a retrospective clinical study on forty-one patients. ANZ J Surg 75:1055-8

17. Fall B, Fall PA, Diao B, et al (2009) Les gangrènes des organes génitaux externes : à propos de 102 cas. Andrologie 19:45-9

18. Hodonou R, Hounnasso PP, Gbessi DG, Akpo C (2000) Les gangrènes pénopérinéoscrotales. Aspects épidémiologiques, diagnostiques et thérapeutiques. À propos de 32 cas. Prog Urol 10:271-6

19. Michel F, Fauchery A, Belhadj M, et al (1997) Apport de l'imagerie dans le diagnostic précoce de la gangrène de Fournier. Prog Urol 7:471-5

20. Unalp HR, Kamer E, Derici H, et al (2008) Fournier's gangrene: evaluation of 68 patients and analysis of prognostic variables. J Postgrad Med 54:102-5

21. Hubert J, Fournier G, Mangin P, Punga-Maole M (1995) Gangrène des organes génitaux externes. Prog Urol 5:911-24

22. De Jong Z, Anaya Y, Pontonnier F, et al (1992) Évolution et traitement de huit malades atteints d'une gangrène périnéoscrotale de Fournier. Ann Urol 26:364-7

23. Sorensen MD, Krieger JK, Rivara FP, et al (2009) Fournier's gangrene: management and mortality predictors in a population based study. J Urol 182:2742-7

24. Gurdal M, Yucebas E, Tekin A, et al (2003) Predisposing factors and treatment outcome in Fournier's gangrene. Analysis of 28 cases. Urol Int 70:286

25. Yilmazlar T, Ozturk E, Ozguc H, et al (2010) Fournier's gangrene: an analysis of 80 patients and a novel scoring system. Tech Coloproctol 14:217-23

26. Berger M (1966) Les antibiotiques. Les grandes heures de la science moderne. Nouveaux horizons, pp 9-13 